\title{
Regression of Atherosclerotic Lesions by High Density Lipoprotein Plasma Fraction in the Cholesterol-fed Rabbit
}

Juan Jose Badimon, Lina Badimon, and Valentin Fuster

Division of Cardiology, The Mount Sinai Medical School of Medicine, New York 10029

\begin{abstract}
The effects of homologous plasma HDL and VHDL fractions on established atherosclerotic lesions were studied in cholesterol-fed rabbits. Atherosclerosis was induced by feeding the animals a $0.5 \%$ cholesterol-rich diet for $60 \mathrm{~d}$ (group 1). Another group of animals were maintained on the same diet for 90 d (group 2). A third group was also fed the same diet for $90 \mathrm{~d}$ but received $50 \mathrm{mg}$ HDL-VHDL protein per wk (isolated from normolipemic rabbit plasma) during the last $30 \mathrm{~d}$ (group 3 ). Aortic atherosclerotic involvement at the completion of the study was $34 \pm 4 \%$ in group $1,38.8 \pm 5 \%$ in group 2 , and $17.8 \pm 4 \%$ in group $3(P<0.005)$. Aortic lipid deposition was also significantly reduced in group 3 compared with group 1 (studied at only $60 \mathrm{~d}$ ) and group 2 . This is the first in vivo, prospective evidence of the antiatherogenic effect of HDLVHDL against preexisting atherosclerosis. Our results showed that HDL plasma fractions were able to induce regression of established aortic fatty streaks and lipid deposits. Our results suggest that it may be possible not only to inhibit progression but even to reduce established atherosclerotic lesions by HDL administration. (J. Clin. Invest. 1990. 85:1234-1241.) atherosclerosis • cholesterol-fed rabbit • HDL-VHDL • regression • lipoprotein
\end{abstract}

\section{Introduction}

The most important characteristic of atherosclerotic arteries is the accumulation of cholesterol within the vessel wall as a consequence of an unbalanced cholesterol influx-efflux. "Reverse cholesterol transport" seems to be the major route for removal of the exchangeable cholesterol deposited in tissues that do not metabolize it, and for its transport to the liver and subsequent metabolization $(1,2)$. The concept of a reverse cholesterol transport pathway, with the HDL particle serving as scavenger of tissue cholesterol, has been enthusiastically accepted since it was postulated by Glomset more than $20 \mathrm{yr}$ ago (3).

The role of plasma HDL as the preferential physiological acceptor for cholesterol from extrahepatic cells in vivo has been strongly supported by epidemiologic and in vitro studies (4-17). Furthermore, we have reported that homologous HDL plasma fraction $(d=1.063-1.25 \mathrm{~g} / \mathrm{ml})$ inhibits the develop-

Address reprint requests to Dr. J. J. Badimon, Division of Cardiology, Box 1030, The Mount Sinai Medical Center, One Gustave L. Levy Place, New York, NY 10029.

Received for publication 12 February 1989 and in revised form 11 October 1989.

J. Clin. Invest.

(C) The American Society for Clinical Investigation, Inc.

0021-9738/90/04/1234/08 \$2.00

Volume 85, April 1990, 1234-1241 ment of fatty streaks and lipid deposition in the aortic wall of cholesterol-fed rabbits (18).

The aim of the present study was to determine whether homologous HDL-VHDL could affect the progression of established atherosclerotic lesions in the cholesterol-fed rabbit. We found that the plasma lipoproteins of high density were able to induce regression of the established aortic fatty streaks and lipid infiltration.

\section{Methods}

Animal model. Adult New Zealand white rabbits $(3.0 \pm 0.5 \mathrm{~kg}$ body $\mathrm{wt})$ were housed in the Animal Research Center of our institution. They were individually caged in stainless-steel wire-bottomed cages, in a room controlled to $20 \pm 2^{\circ} \mathrm{C}$ temperature, $50 \pm 10 \%$ humidity, on a 12 -h light/dark cycle. Our animal care facility is accredited by the American Association for Accreditation of Animal Laboratory Care, and all procedures were reviewed and approved by the Institutional Animal Care and Use Committee.

Atherosclerosis was induced by feeding the animals a $0.5 \%$ cholesterol-rich diet at a daily amount of $140 \mathrm{~g}$. The atherogenic diet was prepared by spraying normal rabbit chow (HF No. 5326; Ralston Purina Company, St. Louis, MO) with cholesterol dissolved in ethyl-ether and allowing the solvent to evaporate in a fume hood. Water was provided ad lib. $(18,19)$

Preparation and isolation of lipoproteins. Lipoproteins were obtained from normal rabbit plasma according to standard procedures (20), adjusting densities by $\mathrm{KBr}$ as previously described $(18,19)$. The lipoprotein fraction selected for our study was obtained at a density range of $1.063-1.25 \mathrm{~g} / \mathrm{ml}$, which includes HDL $(d=1.063-1.21 \mathrm{~g} / \mathrm{ml})$ plus VHDL $(d=1.21-1.25 \mathrm{~g} / \mathrm{ml})$. The HDL-VHDL fraction for injection, in all instances $<4 \mathrm{wk}$ old, was sterilized by filtration through a NALGENE sterilization filter unit type LS with a pore size of $0.2 \mu \mathrm{m}$ (Sybron Corp., Rochester, NY).

Protein content of the HDL-VHDL lipoprotein fraction was determined, after sterilization, by Markwell's modification (21) of Lowry's method in the presence of SDS using bovine serum albumin as standard. The purity of the fraction, namely, the absence of apo B, was tested by SDS polyacrylamide electrophoresis (22)(Fig. 1). The plasma fraction of HDL contained apo A-I, AII, E, and C's. In addition, this plasma fraction has been reported to include the lipid transfer proteins, which enhance transfer and exchange of cholesterol esters, phospholipids, and triglycerides between lipoproteins in plasma (23-27).

The lipid composition per milligram of protein of the administered lipoprotein fraction was $0.2 \pm 0.03 \mathrm{mg}$ cholesterol, $0.1 \pm 0.02 \mathrm{mg}$ triglycerides, and $0.4 \pm 0.05 \mathrm{mg}$ phospholipids as determined by specific enzymatic assays (Dry Stat; Seragen Inc., Boston, MA, and Wako Pure Chemical Industries, Ltd., Dallas, TX, respectively). The administered HDL-VHDL solution had an osmolality of $302 \mathrm{mosmol} /$ liter.

Experimental design. 24 healthy rabbits were selected by body weight and plasma lipid levels into a homogeneous group. Our "homogeneous group" is defined according to the triglycerides and total and HDL-cholesterol levels obtained from a pool of control rabbits, which actually comprises more than 250 healthy animals. As described in Fig. 2 , all the animals were kept on the same atherogenic diet throughout the study. The atherogenic diet consisted of a $0.5 \%$ cholesterol-rich diet given at a daily amount of $140 \mathrm{~g}$. After $60 \mathrm{~d}$ on the atherogenic diet, 
a

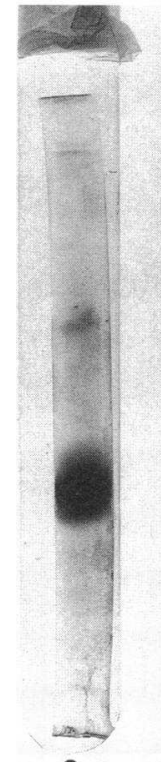

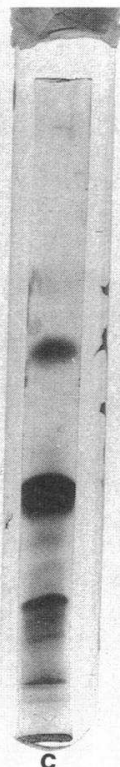

C

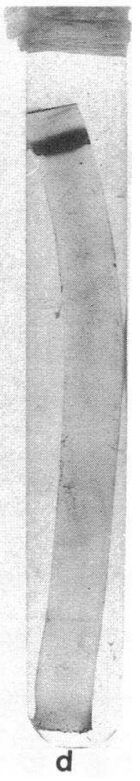

Figure 1. SDS-polyacrylamide electrophoresis. From left to right: (a) standard rabbit apolipoproteins A-I and E; $(b$ and $c$ ) two different concentrations of the HDL-VHDL lipoprotein fraction used in our study; and $(d)$ purified rabbit LDL. The figure shows total absence of apo $B$ in our HDL-VHDL preparation.

group 1, a randomly selected group of 10 animals, were killed and the aortic fatty streak formation and cholesterol deposition were studied. This group provided the baseline for atherosclerotic lesions obtained before the HDL-VHDL or saline administration. Groups $2(n=7)$ and $3(n=7)$ were studied after 30 more days on the atherogenic diet. During this period, group 3 received the HDL-VHDL plasma fraction, while group 2 was kept under the same experimental conditions as group 3 and served as the atherosclerosis-progression control (placebo group). Group 3 received $50 \mathrm{mg}$ of HDL-VHDL protein in saline solution (final volume, $5 \mathrm{ml}$ ), given intravenously via the marginal ear vein once a week during the $4 \mathrm{wk}$. Group 2 was given saline solution on the same schedule and under the same conditions as the treated group (group 3). The animals of these two groups were sacrificed by pairs 1 wk after the last lipoprotein administration.

Morphological and histological evaluation of atherosclerosis. The rabbits were anesthetized with Ketamine plus Rompun ( 5 and 35 $\mathrm{mg} / \mathrm{kg}$, respectively, given intramuscularly). The carotid artery was cannulated, and the animals were exsanguinated. After laparotomy and exposure of the aorta, the animals were killed by an overdose of anesthetic, and the aortas were perfused in situ with $0.2 \mathrm{M}$ phosphatebuffered saline containing papaverine $\left(60 \mathrm{mg} / 500 \mathrm{~cm}^{3}\right)$ at $37^{\circ} \mathrm{C}$ to avoid vasoconstriction. The aortas were removed intact from the aortic arch to the iliac bifurcation and were stripped of all adventitial debris. Thereafter, they were cut lengthwise into two halves through a dorsal and a ventral incision. Care was taken to dissect between the dorsal bifurcations so that the flow dividers were evenly split between the two halves. One half of each aorta was fixed in $10 \%$ buffered formalin, and the other half was processed for lipid analysis. The fixed aortas were stained by Sudan IV to reveal sudanophilic plaques (28). After staining, the aortas were pinned open to flatten them. A template was then made for each aorta by tracing the outlines of the aorta and the atheromatous lesions on a clear plastic sheet. Each template was magnified $(\times 2)$ and photographed. Morphometric assessment of the percentage of total aorta covered with lipid deposits (Sudan-positive area) was determined by computerized planimetry (EM image analyzer; model 124; Numonics Corp., Lansdale, PA). Each of three blinded observers prepared a template for each aorta, and each then performed planimetric measurements on his own templates. Inter-observer variation was $<1 \%$. All measurements were averaged for each aorta.

Since atherosclerotic lesions form at specific sites, such as the bifurcation points of branching vessels, we alternated between choosing the right or left half of each aorta for biochemical or morphological analysis, so as to compensate for differences between the two halves and negate possible unequal sectioning.

For light microscopy sections, segments of the thoracic aortas after Sudan IV staining were embedded in paraffin and stained with hematoxylin-eosin.

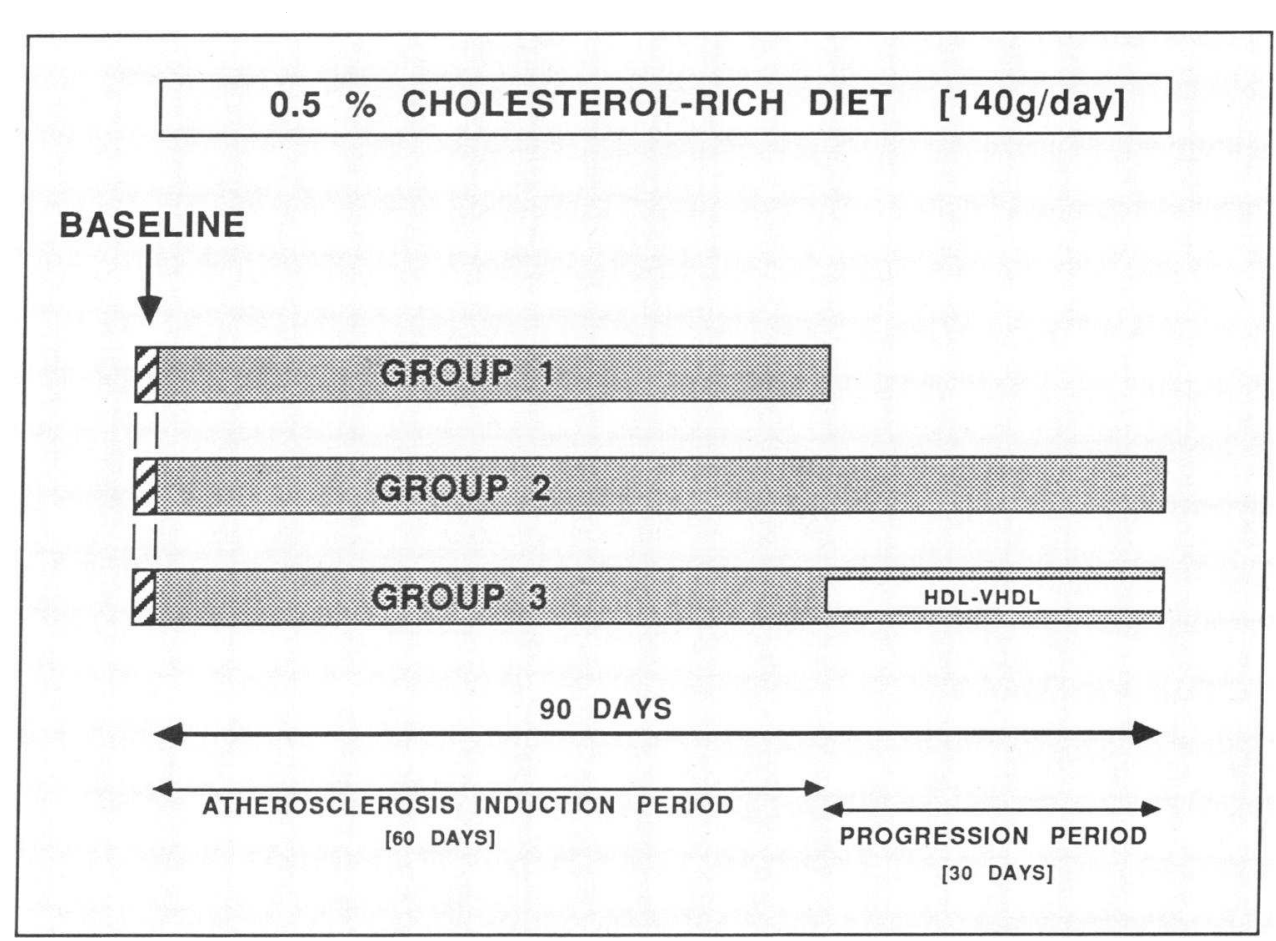

Figure 2. Experimental design of our study. Group 1 was killed after $60 \mathrm{~d}$ on the atherogenic diet and served as a control for the extent of aortic fatty streaks and lipid infiltration obtained before HDL-VHDL administration. Groups 2 and 3 were kept on the atherogenic diet for 30 more days. Group 2 served as a control of atherosclerosis-progression and, since it was kept under the same experimental conditions (receiving saline solution), served as a placebo group. Group 3 was given $50 \mathrm{mg}$ HDL-VHDL protein/week during the last $30 \mathrm{~d}$ (atherosclerosisprogression period). 
Plasma lipid analysis. Blood samples were collected in EDTA (1.5 $\mathrm{mg} / \mathrm{ml}$ ). All the animals were tested for their baseline and killing levels of total and HDL-cholesterol and triglycerides after 12-16 h fasting. Plasma was immediately obtained by low speed centrifugation at $4^{\circ} \mathrm{C}$. Aliquots were separated and kept frozen at $-70^{\circ} \mathrm{C}$ until assayed. Cholesterol content was determined using an enzymatic kit (Dry Stat; Beckman Instruments, Inc., Carlsbad, CA). Triglyceride content of plasma was measured using an enzymatic kit (Seragen Diagnostics Inc., Indianapolis, IN) as previously described $(18,19)$. HDL-cholesterol was measured in plasma after precipitation of apo B-containing lipoproteins by PEG-6000 (29), as we have described $(18,19)$. The HDL-cholesterol was isolated in an aliquot of fresh plasma and thereafter kept frozen until assayed.

Biochemical analysis of aortic wall and liver. Aortas (half) were homogenized at $4^{\circ} \mathrm{C}$ in $5 \mathrm{ml}$ of $0.13 \mathrm{M}$ Tris $\mathrm{HCl}\left(\mathrm{pH} \mathrm{7.4),} 0.01 \% \mathrm{NaN}_{3}\right.$ using a homogenizer (Polytron Corp., Elkhart, IN). The total lipids were extracted from the homogenates in $10 \mathrm{vol}$ of chloroform/methanol $(2: 1 ; \mathrm{vol} / \mathrm{vol})$ containing $0.001 \%$ butylated hydroxytoluene $(2,5-$ Di-t-butyl-p-cresol 4-methyl-2,6-test-butylphenol) as an antioxidant. A second extraction with the Folch procedure was then carried out (30).

The lipid-containing fraction was dried under nitrogen and then resuspended in isopropyl alcohol (31). The triglycerides, total cholesterol, and free cholesterol levels were measured by the specific enzymatic assays mentioned for plasma lipid analysis; esterified cholesterol was calculated as the difference between total and free cholesterol. Phospholipid determinations were performed using an enzymatic kit (Wako Pure Chemical Industries, Ltd.).

The same procedure was followed with the livers. Livers were homogenized, and total cholesterol levels measured in the lipid extracts (31) by using a specific enzymatic assay (Dry Stat; Beckman Instruments, Inc.).

Hematological analysis and animal surveillance. Platelet number, size, and distribution were determined with a P260 platelet analyzer (Coulter Electronics Inc., Hialeah, FL) as we have reported (32). Rabbit platelets and red blood cells are smaller than those of humans, therefore, new thresholds had to be defined in the counter to analyze rabbit blood. After a preliminary study, a lower and upper threshold of 1.73 and $15.7 \mu \mathrm{m}^{3}$, respectively, were determined to include the smallest platelets and to avoid counting the micro-red blood cells as large platelets. Hematocrit was also measured by the microhematocrit technique (Readacrit centrifuge; Clay Adams, Parsippany, NJ).

Animals were closely followed during the experimental period. Their daily food intake was recorded and their weight taken once a week to detect any sudden weight loss.

Statistical analysis. Results are expressed as mean \pm SEM unless otherwise stated. Multiple group means were compared by single or multiple factor analysis of variance (ANOVA) ${ }^{1}$ with factorial analysis and differences between groups analyzed by Fisher protected least significant difference and Scheffe $F$ tests.

\section{Results}

Plasma lipids. All the animals responded to the atherogenic diet with similar, marked increases in total plasma cholesterol compared with prediet values (prediet baseline $=61 \mathrm{mg} / \mathrm{dl}$; $60-\mathrm{d}$ level $=1,559 \mathrm{mg} / \mathrm{dl}$ ) (Fig. 3). At $60 \mathrm{~d}$, group 1 animals were killed. The remaining animals were randomly divided into two groups and continued on the atherogenic diet for $\mathbf{3 0}$ more days. Plasma cholesterol levels obtained in groups 2 and 3 at $90 \mathrm{~d}$ were not significantly different from those obtained at $60 \mathrm{~d}$. There were no differences in total plasma cholesterol, HDL-cholesterol, or triglycerides between groups 2 and 3 (Fig. 3) after $90 \mathrm{~d}$ on the high-cholesterol diet.

1. Abbreviation used in this paper: ANOVA, analysis of variance.
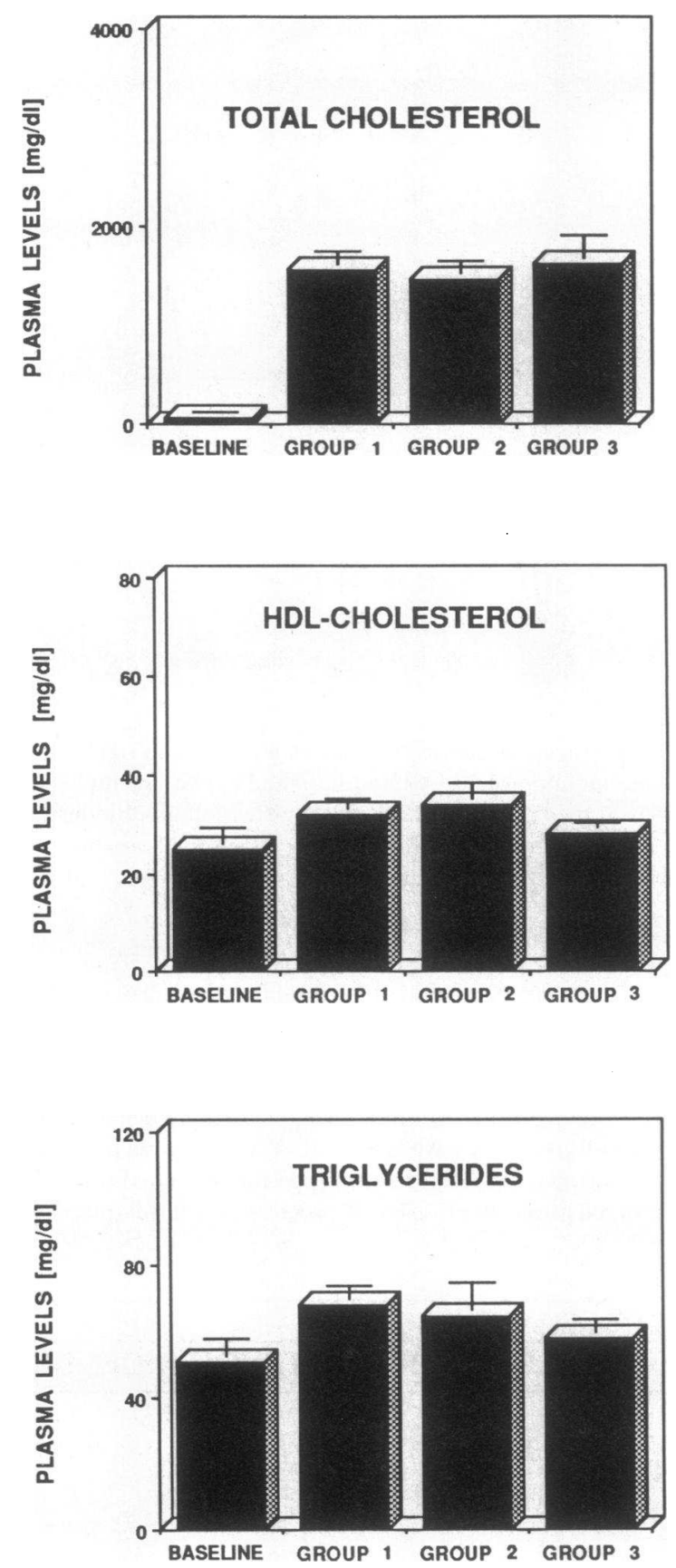

Figure 3. Plasma levels of total cholesterol, HDL-cholesterol, and triglycerides. Values are expressed as milligram per deciliter; mean $\pm \mathrm{SE}$ Baseline corresponds to all the prediet values of all the animals included in our study. Group 1 animals were kept for $60 \mathrm{~d}$ on the atherogenic diet (atherosclerosis-induction control). Group 2 animals were kept for $60 \mathrm{~d}$ on the atherogenic diet plus 30 more days on the same diet while receiving saline solution (atherosclerosis progression control). Group 3 animals were kept $60 \mathrm{~d}$ on the atherogenic diet plus 30 more days on the same diet while receiving HDL-VHDL.

At $90 \mathrm{~d}$, total cholesterol distribution among the different lipoprotein subfractions was similar in both groups (Table I). The so-called "atherogenic" lipoproteins (VLDL and LDL; $d$ $<1.063 \mathrm{~g} / \mathrm{ml}$ ) accounted for more than $95 \%$ of the total cholesterol. All groups showed a marked decrease in the choles- 
Table I. Total Cholesterol Distribution among Plasma Lipoproteins

\begin{tabular}{lcc}
\hline & Group 2 & Group 3 \\
\hline VHDL & $902 \pm 178$ & $811 \pm 100$ \\
LDL & $407 \pm 61$ & $398 \pm 37$ \\
HDL & $38 \pm 18$ & $37.5 \pm 21$ \\
\hline
\end{tabular}

Results expressed as milligram per deciliter (mean $\pm \mathrm{SE}$ ). Blood samples were obtained at the end of the experiments, $1 \mathrm{wk}$ after the last HDL-VHDL administration.

terol carried by the HDL fraction from $43 \%$ at baseline to $3 \%$ at killing.

Morphological and histological analysis. Group 1 (atherosclerosis control group) were killed $60 \mathrm{~d}$ after beginning the atherogenic diet and served as a reference point for the level of atherosclerotic lesions achieved in the other 2 groups before the HDL or saline administration. Groups 2 and 3 were kept on the same diet for 30 more days and then killed. When the three groups (groups 1,2, and 3) were analyzed for the presence of aortic lipid-rich lesions (fatty streaks), all groups showed the atherosclerotic lesion distribution and characteristics typical of this experimental model. Lesions consisted of fatty streaks, affecting mainly the aortic arch and the descending thoracic aorta. The bifurcation points, such as the intercostal ostia, were affected most; the abdominal aorta much less so.

The aortic atherosclerotic involvement was evaluated by computerized planimetry of the Sudan IV-positive areas, expressing the results as a percentage of the total aortic surface covered by fatty lesion (Fig. 4). Group 1 ( $60 \mathrm{~d}$ on atherogenic diet) showed $34 \pm 4 \%$ of the aortic surface affected. After 30 more days on the cholesterol-rich diet, the aortic surface of group 2 ( $90 \mathrm{~d}$ on atherogenic diet) was $38.8 \pm 5 \%$ involved. The results showed a trend toward greater aortic atherosclerotic involvement, as a consequence of the longer period of atherogenic diet ingestion, even though it did not reach statistical significance. In contrast, group 3 (90 d on diet plus HDLVHDL) had only $17.8 \pm 4.1 \%$ of their aortic surface covered by

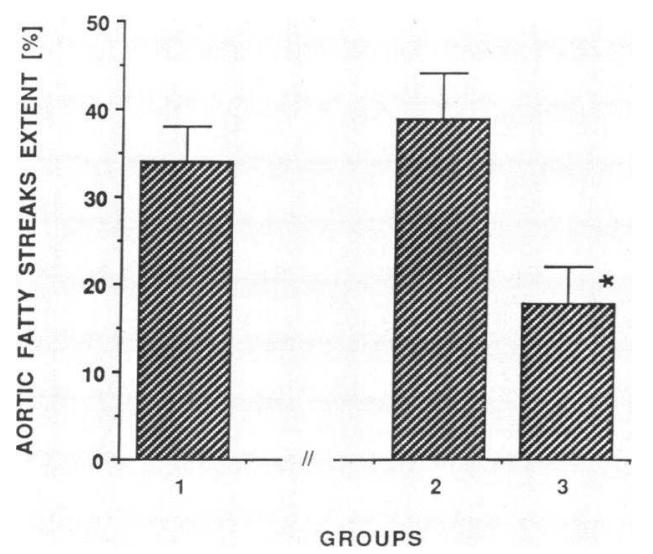

Figure 4. Aortic atherosclerotic involvement. Values are expressed as $\% \pm$ SE of aortic surface covered by fatty streaks. Analysis of data by ANOVA $\left({ }^{*}, P<0.05\right.$ compared with both groups 1 and 2$)$. fatty streaks, a statistically significant difference $(P<0.005)$ not only compared with group 2 (90 d on diet), but also when compared even with group 1 (60 d on diet) $(P<0.005)$.

Histological examination of the aortas of the three groups of animals revealed similar lesions characterized by accumulation of lipids in the hyperplastic areas of the inner layer of the intima (Fig. 5). A decrease in lesion thickness in the group 3 (HDL-VHDL) animals (Fig. $5 A$ ) is evident in comparison with the animals in groups 1 and 2 (Fig. $5, B$ and $C$, respectively).

Lipid accumulation in the aortic wall. To analyze lipid infiltration, we performed a chemical analysis of the lipids extracted from the aortic wall. Values of total cholesterol, free cholesterol, esterified cholesterol, triglycerides, and phospholipids accumulated within the aortic wall are presented in Fig. 6.

Group 2 (90 d on diet) showed heavier lipid deposition in the aortic wall than group 1 (atherosclerosis-induction $60 \mathrm{~d}$ on diet), probably due to the longer time on the diet. Group 3 (HDL-treated) had a 55\% reduction of total cholesterol $(P$ $<0.05), 59 \%$ reduction of esterified cholesterol $(P<0.05)$, and $59 \%$ reduction of phospholipids $(P<0.05)$ with no differences in free cholesterol and triglycerides when compared with group 2 . When group 3 was compared with group 1 (only $60 \mathrm{~d}$ on atherogenic diet) significant decreases in total cholesterol $(P$ $<0.05)$, free cholesterol $(P<0.05)$, and phospholipids $(P$ $<0.05$ ) were also observed (Fig. 6).

Cholesterol accumulation in the liver. The total cholesterol content in the livers is presented in Fig. 7. HDL-VHDL treatment (group 3) showed a trend toward lesser cholesterol accumulation in the livers even though it did not reach statistical significance.

Effect of diet and treatment. All groups had similar body weight gain throughout the experimental period. The HDLVHDL-treated rabbits gained an average of $0.85 \pm 0.4 \mathrm{~kg}$ (range $0.5-1.0 \mathrm{~kg}$ ), and group 2 gained an average of $0.7 \pm 0.2$ $\mathrm{kg}$ (ranging from 0.4 to $0.8 \mathrm{~kg}$ ). No difference in food intake was observed among groups. Hematocrit, platelet, and red blood cell counts were similar in all groups. The animals showed good physical appearance and normal behavior during the entire experiment.

\section{Discussion}

In a previous study we have shown that homologous HDLVHDL administered concomitantly with an atherogenic diet inhibits aortic fatty streak formation and cholesterol deposition in cholesterol-fed rabbits (18). The present study sought to determine if homologous HDL-VHDL administration, after atherosclerotic lesions have been established, could inhibit or reverse its further development. After the high-cholesterol diet was given for $60 \mathrm{~d}$, the animals had $34 \%$ of their aortic surface covered by fatty streaks containing $15 \pm 2.4 \mathrm{mg}$ of cholesterol/g of aorta. These values match those of previous studies using the same dietary method of atherosclerosis induction $(18,19)$. The remaining animals of the study were kept on the same diet for 30 more days with one group (group 3), being given 50 $\mathrm{mg} / \mathrm{wk}$ of homologous HDL-VHDL protein and the other (group 2) given saline solution as control.

Our results showed that administration of homologous HDL-VHDL not only inhibited the progression of established 
A

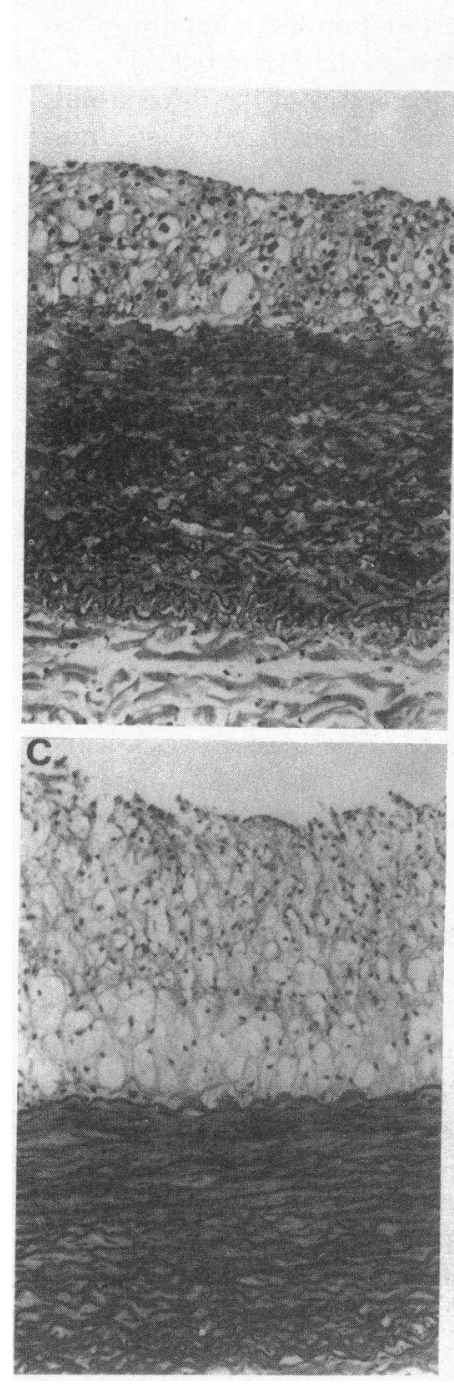

B

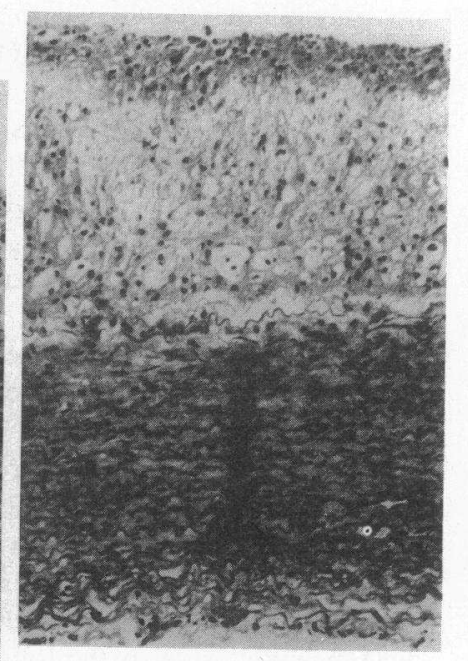

D

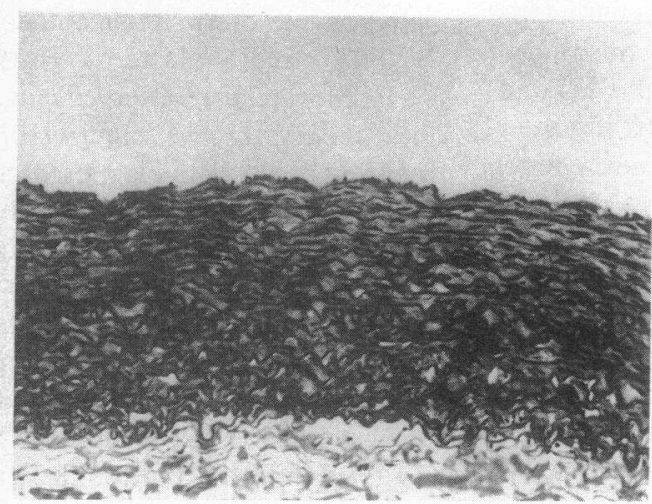

Figure 5. Representative aortic sections from HDL-treated $(A)$, placebo $(B)$, atherosclerotic-control $(C)$, and normal $(D)$ rabbits. Sections were taken from similar areas of the thoracic aorta. Normal animals were fed a standard rabbit chow. (hematoxylin-eosin stain, $\times 80$ ). atherosclerotic lesions, when compared with group 2 ( $90 \mathrm{~d}$ on diet), but also induced significant regression of aortic fatty streaks and cholesterol deposition when compared with group 1 (atherosclerosis-induction group, $60 \mathrm{~d}$ on diet). Therefore, HDL-VHDL have been shown to reverse the extent of arterial damage and lipid infiltration induced by $90 \mathrm{~d}$ of an atherogenic diet to levels significantly lower than those achieved after $60 \mathrm{~d}$ on the diet. HDL-treated animals (group 3) developed $54 \%$ less aortic infiltration with fatty streaks, as determined by computerized planimetry of the aortic wall, than the control animals (group 2). Their aortas also contained $56.3 \%$ less cholesterol than those of controls.

An abundance of data confirming the inverse relationship between HDL and arterial atherosclerotic disease have been reported (4-9). To date, such studies have been retrospective, and no prospective interventional studies other than ours (18) have been conducted to test the efficacy of HDL in preventing or inhibiting the development of atherosclerotic disease. Studies in tissue culture systems have lent credence to the hypothesis that HDL protects against the development of atherosclerosis (10-17). Such studies have indicated that HDL may exert this beneficial effect by facilitating reverse cholesterol trans- port, namely, transporting cholesterol away from extra-hepatic tissues and carrying it back to the liver where it can be eliminated (14-16).

Using in vitro systems, other authors have reported that not only HDL, but also VHDL, may exert an antiatherogenic effect $(14,16,33)$. For example, plasma proteins obtained in the $d>1.21 \mathrm{~g} / \mathrm{ml}$ fraction can remove cholesterol from macrophages $(14,33)$, while albumin and $\gamma$-globulins, the two major proteins of this fraction, did not accept large amounts of cholesterol (14). In our experimental protocol, we used the plasma fraction obtained at density range $1.063-1.25 \mathrm{~g} / \mathrm{ml}$, which contains $\mathrm{HDL}_{2}(d=1.063-1.12 \mathrm{~g} / \mathrm{ml}) \mathrm{HDL}_{3}(d=1.12-1.21$ $\mathrm{g} / \mathrm{ml})$, and VHDL $(d=1.21-1.25 \mathrm{~g} / \mathrm{ml})$. In addition, it has been reported that this density fraction includes the lipid transfer proteins which have been shown to enhance transfer and exchange of lipids between lipoproteins in plasma (23-27). The dosage and frequency of the lipoprotein administration $(50 \mathrm{mg}$ protein content given intravenously once a week), is the same that successfully inhibited the development of fatty streak and lipid accumulation in the aortic wall in our earlier study (18).

In the present study, as expected, all the animals rapidly 

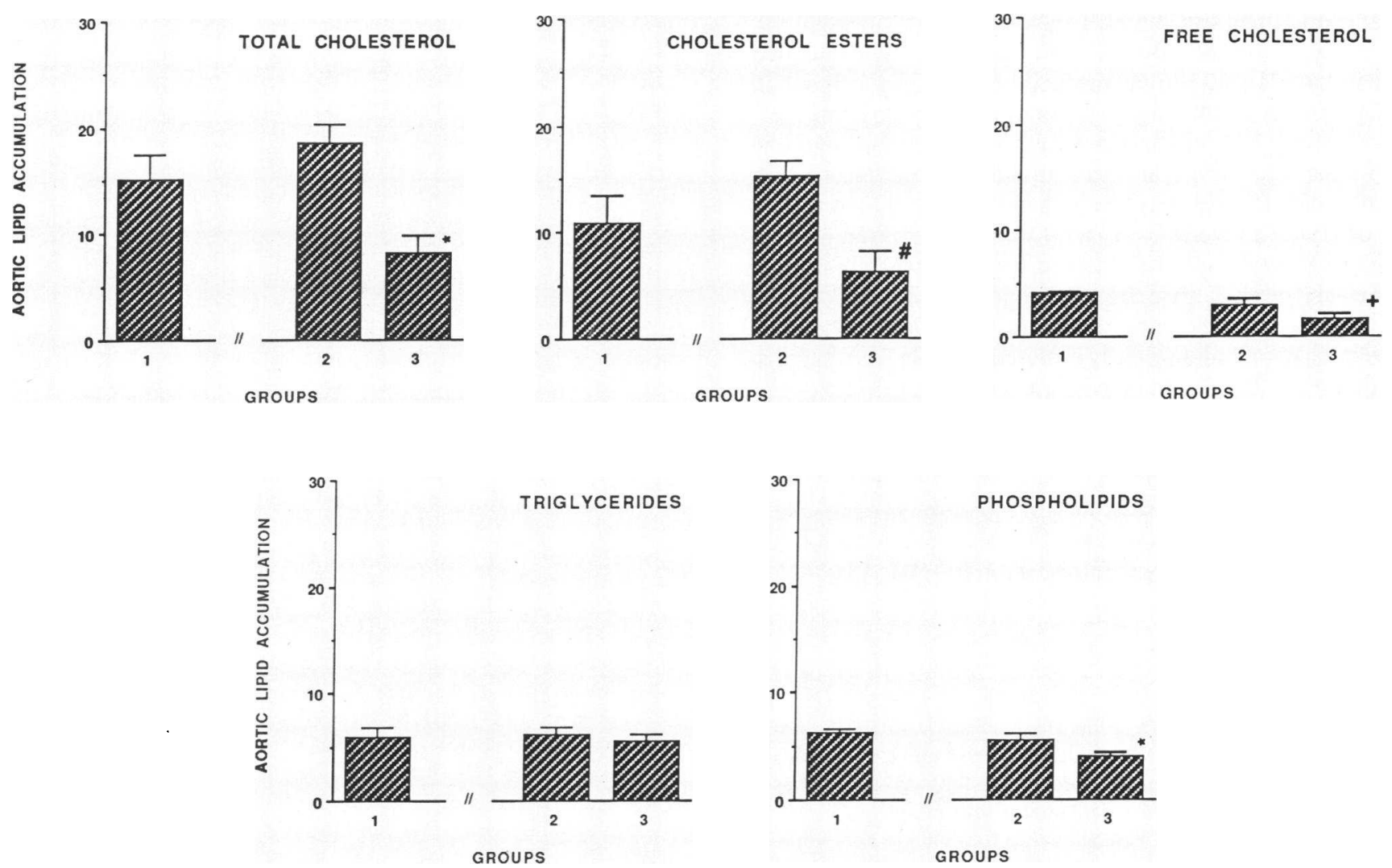

Figure 6. Aortic lipid accumulation. Values are expressed as $\mathrm{mg} / \mathrm{g}$ dry tissue (mean $\pm \mathrm{SE}$ ). Data analysis by ANOVA $\left({ }^{*}, P<0.05\right.$ compared with groups 1 and $2 ;+, P<0.05$ compared with group 1 ; ", $P<0.05$ compared with group 2 ).

developed hypercholesterolemia upon atherogenic diet ingestion. No statistically significant difference in plasma lipids was observed between the groups. Cholesterol distribution among the different lipoproteins showed that apo-B containing lipoproteins $(d<1.063 \mathrm{~g} / \mathrm{ml})$ accounted for $\sim 95 \%$ of the total plasma cholesterol, being the ratio of HDL-cholesterol to total cholesterol markedly reduced in comparison with prediet levels.

HDL-VHDL treatment induced a significant reduction in the extent of aortic atherosclerotic involvement when compared with groups 1 and 2 . Because plasma lipid levels were not modified by the treatment, there seems to be a direct role for HDL in aortic cholesterol metabolism. Similar inhibition of cholesterol-induced atherosclerosis without change in plasma lipid levels has been observed in rabbits given estrogens

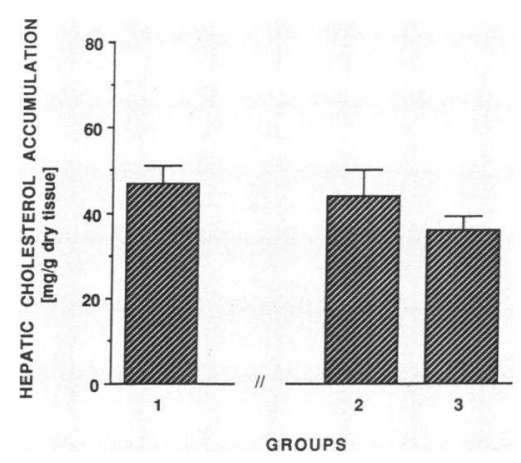

Figure 7. Hepatic accumulation of total cholesterol. Values are expressed as $\mathrm{mg} / \mathrm{g}$ dry tissue (mean $\pm \mathrm{SE})$.
(34), verapamil (35), a specific thromboxane $A_{2}$ synthetase inhibitor (36), polyunsaturated oils (37), and probucol (38, $39)$. It has also been reported that intravenous administration of large amounts of dispersed phospholipids (0.5-3.5 $\mathrm{g}$ two to three times a week) induces regression of experimental atherosclerosis in rabbits (39-41).

In a preliminary study, we reported that HDL stimulated prostacyclin $\left(\mathrm{PGI}_{2}\right)$ synthesis by aortic wall in normal (42) and atherosclerotic rabbits (43). It has been reported that prostaglandins modulate arterial cholesteryl-ester metabolism by stimulating the enzyme cholesteryl-hydrolase (44). Therefore, an increased prostacyclin production in the HDL-treated group might also account for the observed antiatherogenic effect.

It has also been shown that HDL reverses the accumulation of cholesteryl esters by continuous hydrolysis and reesterification in macrophage-like cell lines (45).

Of interest is the observation that the HDL-VHDL-treated group (group 3) presented a lesser extent of aortic fatty streaks (Sudan IV-positive areas) than did group 1 (atherosclerosisinduction group) (Fig. 4). A similar finding was observed when analyzing the lipid accumulation within aortic wall (Fig. 6). HDL-VHDL treatment not only significantly inhibited aortic lipid deposition when compared with group 2 , but also yielded a significantly lower lipid accumulation than in group 1. Inasmuch as all the animals had similar levels of plasma cholesterol available for deposition within vessel wall, our results strongly support the hypothesis that homologous HDL-VHDL administration stimulates reverse cholesterol transport from 
extrahepatic tissues to the liver, where it can be metabolized and excreted. In addition, as shown in Fig. 5, HDL-treated animals have less subintimal proliferation than groups 1 and 2 .

Our results parallel findings obtained in tissue culture experiments (10-17) and support for the first time in vivo, the hypothesis that HDLs have an antiatherogenic effect. Such antiatherogenic effect may be mediated through the postulated role of HDL in reverse cholesterol transport, even though no conclusive proof could be obtained from our study and more studies are needed. Regression of atherosclerotic lesions has been reported by different interventions in animal models (see references 46-48) and recently in human studies (49), suggesting that a mechanism may exist for removing the cholesterol accumulated in the vessel wall. The current report is the first prospective in vivo evidence of the inhibitory effect of HDL in established atherosclerosis.

In conclusion, our results suggest that it may be possible to diminish the progression and even reverse already established atherosclerotic lesions by administration of HDL-VHDL.

\section{Acknowledgments}

The authors wish to thank Dr. Efim Mogilevsky, Dr. Liviu Rappaport, and Magda Pena for expert technical assistance, and Carmen Busnadiego for the preparation of the manuscript. Editorial review was by Karen Sadock, American Medical Writers Association.

Dr. J. J. Badimon is a Chait Scholar in Cardiology. This work was supported by National Institutes of Health grant HL-39840 and by funds from a Heart Research Foundation grant.

\section{References}

1. Reichl, D., and N. E. Miller. 1986. The anatomy and physiology of reverse cholesterol transport. Clin. Sci. (Lond.). 70:221-231.

2. Phillips, M. C., W. J. Johnson, and G. H. Rothblat. 1987. Mechanisms and consequences of cellular cholesterol exchange and transfer. Biochim. Biophys. Acta. 906:223-276.

3. Glomset, J. A. 1968. The plasma lecithin:cholesterol acyltransferase reaction. J. Lipid Res. 9:155-167.

4. Barr, D. P., E. M. Russ, and H. A. Eder. 1951. Protein-lipid relationship in human plasma II. In atherosclerosis and related conditions. Am. J. Med. 11:480-487.

5. Gordon, D., J. L. Probstfield, R. J. Garrison, J. D. Neaton, W. P. Castelli, J. D. Knoke, D. R. Jacobs, S. Bangdiwala, and H. A. Tyroler. 1989. High-density lipoprotein cholesterol and cardiovascular disease. Circulation. 79:8-15.

6. Miller, N. E., O. M. Forde, and S. D. Thelle. 1977. The Tromso heart study: high density lipoproteins and coronary heart disease; a prospective case-control study. Lancet. i:965-968.

7. Rhoads, G. G., C. L. Gulbrandsen, and A. Kagen. 1976. Serum lipoproteins and coronary heart disease in a population study of $\mathrm{Ha}-$ waii Japanese men. N. Engl. J. Med. 294-298.

8. Gordon, T., W. P. Castelli, and M. C. Hjortland. 1977. High density lipoproteins as a prospective factor against coronary heart disease. The Framingham Study. Am. J. Med. 62:707-714.

9. Gordon, T., W. B. Kannel, W. P. Castelli, and T. R. Dawber. 1981. Lipoproteins, cardiovascular disease and death. The Framingham study. Arch. Intern. Med. 14:1128-1135.

10. Jackson, R. L., A. M. Gotto, O. Stein, and V. Stein. 1971. A comparative study on the removal of cellular lipids from Landschut ascites cell by human plasma lipoproteins. J. Biol. Chem. 250:72047209.

11. Stein, O., J. V. Vanderhoek, and Y. Stein. 1977. Cholesterol- ester accumulation in cultured aortic muscle cells. Atherosclerosis. 26:465-482.

12. Stein, O., and Y. Stein. 1976. High density lipoproteins reduce the uptake of low density lipoproteins by human endothelial cells in culture. Biochim. Biophys. Acta. 431:363-368.

13. Bates, S. R. 1980. Effect of HDL on the interaction of hyperlipidemic LDL with monkey smooth muscle cells. Artery. 7:303-308.

14. Ho, Y., M. S. Brown, and J. L. Goldstein. 1980. Hydrolysis and excretion of cytoplasmic cholesteryl-esters by macrophages: stimulation by high density lipoproteins and other agents. $J$. Lipid Res. 21:319-398.

15. Reichl, O., O. N. Rudura, N. B. Myant, and J. Pflig. 1982. Further evidence for the role of high density lipoproteins in the removal of tissue cholesterol in vivo. Atherosclerosis. 44:73-82.

16. Oram, J. F. 1983. Effects of high density lipoprotein subfractions on cholesterol homeostasis in human fibroblasts and arterial smooth muscle cells. Arteriosclerosis. 3:420-432.

17. Hessler, J. R., A. I. Robertson, and G. M. Chisolm. 1979. LDL-induced cytotoxicity and its inhibition by HDL in human vascular smooth muscle and endothelial cells in culture. Atherosclerosis. 32:213-219.

18. Badimon, J. J., L. Badimon, A. Galvez, and V. Fuster. 1989. High density lipoprotein plasma fractions inhibit aortic fatty streak lesions in cholesterol-fed rabbits. Lab. Invest. 60:455-461.

19. Arad, V., J. J. Badimon, L. Badimon, V. C. Hembree, and H. N. Ginsberg. 1989. Dehydroepiandrosterone feeding prevents atherosclerosis in the cholesterol-fed rabbit. Arteriosclerosis. 9:159-166.

20. Havel, R. J., H. S. Eder, and J. M. Bragdom. 1955. The distribution and chemical composition of ultracentrifugally separated lipoproteins in human serum. J. Clin. Invest. 34:1345-1353.

21. Markwell, M. A. K., S. M. Haas, L. L. Bieber, and N. E. Tolbert. 1978. A modification of the Lowry procedure to simplify protein determination in membrane and lipoprotein samples. Anal. Biochem. 87:206-210.

22. Weber, K., and M. Osborn. 1969. The reliability of molecular weights determination by dodecylsulfate polyacrylamide gel electrophoresis. J. Biol. Chem. 244:4406-4412.

23. Morton, R. E., and O. B. Zilversmit. 1982. Purification and characterization of lipid transfer protein(s) from human lipoproteindeficient plasma. J. Lipid Res. 23:1058-1067.

24. Tall, A. R., S. Krumholz, T. Olivercrona, and R. J. Deckelbaum. 1985. Plasma phospholipid transfer protein enhances transfer and exchanges of phospholipids between very low density lipoproteins and high density lipoproteins during lipolysis. J. Lipid Res. 26:842851.

25. Zilversmit, D. 1984. Lipid transfer proteins. J. Lipid Res. 25:1563-1569.

26. Son, Y. S. C., and D. Zilversmit. 1986. Increased lipid transfer activities in hyperlipidemic rabbits plasma. Arteriosclerosis. 6:345351 .

27. Stein, O., G. Halperin, and Y. Stein. 1986. Cholesterol ester efflux from extracellular and cellular elements on arterial wall. Model systems in culture with cholesteryl linoleyc ether. Arteriosclerosis. 6:70-78.

28. Holman, R. L., H. C. McGill, J. P. Strong, and J. C. Gear. 1958. Technics for studying atherosclerotic lesions. Lab. Invest. 7:42-47.

29. Izzo, C., F. Grillo, and F. Murador. 1981. Improved method for determination of high density lipoprotein cholesterol. Isolation of high density lipoproteins by use of polyethylene glycol-6000. Clin. Chem. 27:371-374.

30. Folch, J., M. Lees, and G. H. Sloane Stanley. 1956. A simple method for the isolation and purification of total lipids from animal tissues. J. Biol. Chem. 226:497-509.

31. Naito, H. K., and J. A. David. 1981. Laboratory considerations: determination of cholesterol, triglycerides, phospholipids, and other lipids in blood and tissues. In Laboratory and Research Methods in Biology and Medicine: Lipid Research Methodology. Alan R. Liss, Inc., New York. 1-76. 
32. Galvez, A., L. Badimon, J. J. Badimon, and V. Fuster. 1986. Electrical aggregometry in whole blood from human, pig and rabbit. Thromb. Haemostasis. 56:128-132.

33. Stein, O., Y. Stein, M. Lefevre, and P. S. Roheim. 1986. The role of apolipoproteins A-IV in reverse cholesterol transport studied with cultured cells and liposomes derived from an ether analog of phosphatidylcholine. Biochim. Biophys. Acta. 878:7-13.

34. Hough, J., and D. Zilversmit. 1986. Effect of 17-Beta estradiol on aortic cholesterol content and metabolism in cho-fed rabbits. Arte riosclerosis. 6:57-63.

35. Rouleau, J. L., W. W. Parmley, J. Stevens, J. Wikman-Coffelt, R. Sievers, R. W. Mahley, R. J. Havel, and W. Brecht. 1983. Verapamil supresses atherosclerosis in cholesterol-fed rabbits. J. Am. Coll. Cardiol. 16:1453-1460.

36. Skrinska, V. A., M. Konieczkowski, R. G. Gerrity, C. F. Galang, and M. V. Rebec. 1988. Suppression of foam cell lesions in hypercholesterolemic rabbits by inhibition of thromboxane $A_{2}$ synthesis. Arteriosclerosis. 8:359-367.

37. Leth-Espersen, P., S. Steender, H. Rawn, and K. Kjeldsenk. 1988. Antiatherogenic effect of olive and corn oils in cholesterol-fed rabbits with the same plasma cholesterol levels. Arteriosclerosis. 8:281-287.

38. Carew, T. E., O. L. Schwenke, and D. Steinberg. 1987. Antiatherogenic effect of probucol unrelated to its hypercholesterolemic effect: evidence that antioxidants in vivo can selectively inhibit low density lipoprotein degradation in macrophage-rich fatty streaks and slow the progression of atherosclerosis. Proc. Natl. Acad. Sci. USA. 84:7725-7729.

39. Steinberg, D., S. Pathasarthy, and T. W. Carew. 1988. In vivo inhibition of foam cell development by probucol in Watanabe rabbits. Am. J. Cardiol. 62:6B-11B.
40. Friedman, M., S. O. Byers, and R. N. Rosenman. 1957. Resolution of aortic atherosclerotic infiltration in the rabbit by phosphatide infusion. Proc. Soc. Exp. Biol. Med. 95:586-588.

41. Williams, K. J., V. P. Werth, and J. A. Wolpp. 1984. Intravenously administered lecithin liposomes: a synthetic antiatherogenic lipid particle. Perspect. Biol. Med. 27:417-431.

42. Badimon, L., J. J. Badimon, A. Galvez, and V. Fuster. 1985 Stimulatory effect of high density lipoprotein on vessel wall prostaglandin synthesis. Circulation. 72:1120A. (Abstr.)

43. Badimon, L., J. J. Badimon, A. Galvez, J. Chesebro, and V. Fuster. 1986. Effect of high density lipoproteins on atherosclerotic vessel wall prostaglandin synthesis in vivo. Clin. Res. 34:625A. (Abstr.)

44. Hajjar, D. P. 1984. Prostaglandins modulate arterial cholesteryl ester metabolism. Enzyme (Basel). 32:218-227.

45. Via, D. P., A. L. Plant, I. F. Craig, A. M. Gotto, and L. C. Smith. 1985. Metabolism of normal and modified low density lipoproteins by macrophage cell lines of murine and human origin. Biochim. Biophys. Acta. 833:417-428.

46. Malinow, M. R. 1983. Experimental models of atherosclerosis regression. Atherosclerosis. 48:105-118.

47. Malinow, M. R. 1986. Regression and resolution of atherosclerosis. In Recent Advances in Arterial Disease: Atherosclerosis, Hypertension and Vasospasm. Alan R. Liss, Inc., New York. 31-38.

48. St. Clair, R. W. 1983. Atherosclerosis regression in animal models: current concepts of cellular and biochemical mechanisms. Prog. Cardiovasc. Dis. 26:109-132.

49. Blankenhorn, D. H., S. A. Nessim, R. L. Johnson, M. E. San Marco, S. P. Azen, and L. Cashin-Hemphill. 1987. Beneficial effects of colestipol-niacin therapy on coronary atherosclerosis and coronary venous bypass grafts. JAMA (J. Am. Med. Assoc.). 257:3233-3240. 